\title{
Less Axillary Lymphadenectomy is More Beneficial: 27-Year Follow-up of Patients with Breast Cancer
}

\author{
Seyedmohammadreza Javadi (iD) ${ }^{1,2}$, Mohammad Esmaeil Akbari (iD) ${ }^{2,}$, Solmaz Hashemi (iD ${ }^{3}$, Farid \\ Moradian (iD ${ }^{2}$, Atieh Akbari (iD ${ }^{2}$, Farzane Mohamadi ${ }^{2}$ and Maryam Khayamzadeh (iD ${ }^{2}$ \\ ${ }^{1}$ General Surgery Department, Hamadan University of Medical Sciences, Hamadan, Iran \\ ${ }^{2}$ Cancer Research Center, Shahid Beheshti University of Medical Scienses, Tehran, Iran \\ ${ }^{3}$ General surgery Department,Tabriz Univesity of Medical Sciences, Tabriz, Iran \\ "Corresponding author: Cancer Research Center, Shahid Beheshti University of Medical Scienses, Tehran, Iran. Email: profmeakbari@gmail.com
}

Received 2020 August 16; Revised 2020 November 29; Accepted 2020 December 01.

\begin{abstract}
Background: One of the most important alterations in breast cancer treatment is the change of view in axillary lymph node management. At the moment, sentinel lymph node biopsy (SLNB) is the standard care in axillary lymph node management. However, in patients with clinically positive lymph nodes or in patients, who have no willingness to receive radioactive drugs, axillary lymph node dissection (ALND) must be done. To the best of our knowledge, there is no overall survival (OS) benefit in ALND, especially at the early stage of breast cancer, during which this procedure is not justified.

objectives: Herein, we have reported the results of 27 years of experiments in limited axillary lymph node dissection (LALND) in comparison to ALND as well as the relationship among the number of removed lymph nodes, OS, and disease-free survival (DFS) at the early stage of breast cancer.

Methods: OS and DFS for 588 cases, who were at the early stage of breast cancer and treated by LALND between 1984 and 2019 , were compared with 1026 patients, who were treated by ALND during the same interval in this study. Notably, SLNB cases were excluded. Results: The results revealed no significant difference among the groups in terms of DFS ( $P=0.268,0.123$, and 0.333$)$. Also, there was no difference in terms of OS between the LALND group (1 - 4 nodes, 5 - 6 nodes, and 7 - 8 nodes) and ALND group ( $\geq 9$ nodes) in patients without lymph node involvement (AHR less than 2). However, in the patients with axillary lymph node metastasis $\left(\mathrm{N}_{1}, \mathrm{~N}_{2}\right.$ ), similar results were obtained. Correspondingly, in this group, the best results were observed in those patients, whose 7 - 8 lymph nodes were removed.

Conclusions: Regarding the results of the current study; it can be concluded that performing the LALND in the defined anatomic range and removing 7-8 lymph nodes instead of removing 10 lymph nodes are not inferior when it is not possible to do SLNB (there is no access to it) and/or being a contraindication to do it for evaluating the status of axillary lymph nodes in the patients at the early stage of breast cancer.
\end{abstract}

Keywords: Breast Neoplasms, Lymph Node Excision, Sentinel Lymph Node Biopsy

\section{Background}

Axillary lymph nodes are known as the most common places of metastasis in breast cancer (1). Nowadays, breast cancer can be mainly treated through surgery, radiotherapy, and systemic therapy. From the beginning era of using surgery for breast cancer, the removal of axillary lymph nodes has been known as an integral part of breast surgery and its therapeutic role has been considered for lymph nodes surgery. Galan, who was a Greek physician and surgeon (210 - 129 B.C), had first revealed the systematic nature of breast cancer. Accordingly, he believed that this disease results from an increase in black bile. Avicenna believed that surgery is the treatment at the initial stages; he was also the first one who stated that in his book "Qanoon", breast cancer is related to axillary lymph nodes (2). For several centuries, surgery was recommended as an effective treatment for limited diseases, and non-surgery treatments were recommended for advanced diseases. In the 1900s, William Halsted introduced a new surgery method called radical mastectomy after conducting a non-randomized controlled clinical trial, which showed local control of the disease. However, no change was observed in overall survival (OS). Halsted and his followers removed all axillary lymph nodes including levels 
I, II, and III $(3,4)$. In later modifications of surgery, axillary surgery was only limited to levels I and II along with some limitations in breast surgery. The increased use of radiotherapy, as an adjuvant treatment, resulted in the success of this method. However, some individuals still prefer wide-range dissection with no radiotherapy until the early 1990s.

Sandowski et al. (1991) in their study revealed that total axillary dissection with no radiotherapy is accompanied by a high local control and a low complication; so, this method is better than dissection levels I and II along with radiotherapy (5). However, some later studies have shown that OS is lower in the cases with more than 20 removed lymph nodes compared to those cases with less than 20 removed lymph nodes (86\% vs 96\%) (6). In 2005, the SEER study on 257157 patients showed that removing more than 4 lymph nodes can lead to better survival (7).

In another study conducted at a lower scale, it was shown that removing more than 10 lymph nodes is not accompanied by better survival in those patients, whose lymph nodes were not involved (8). Thus, its therapeutic role is not currently considered for axillary lymph node dissection. Besides, the involvement of these nodes is a valuable prognostic factor $(1,8)$. Moreover, predicting the chance of being a cancer cell for lymph nodes is very important. In this regard, in a study conducted by Akbari et al., their results showed that tumor size is an important factor in predicting the involvement of lymph nodes, in which the bigger the size of the tumor showed more chance of metastasis into lymph nodes (9). Accordingly, similar studies confirmed these results (10).

Realizing the lymphatic flow of the breast is very important to realize the metastasis of cancer cells into lymph nodes. In a study, Veronesi showed that lymphatic flow from breast to axilla is firstly drained to level I, then to level II, and finally to level III. Also, the chance of skip lesion was estimated to be about $2 \%$, which is lower than 2 in 1000 for level III $(1,11,12)$. This realization and the use of adjuvant treatments including chemotherapy (ChT) and radiotherapy (RT) were introduced and, then, developed the limited axillary surgery, especially at the early stage of breast cancer.

The results of some randomized controlled trials on local therapy of breast cancer done in Cardiff revealed no differences in terms of disease-free survival (DFS) and OS between axillary sampling patients with total axillary dissection (13). Furthermore, in a randomized trial, Forrest et al. observed no difference in terms of DFS and OS between early stage breast cancer patients, who underwent limited axillary dissection patients with total axillary dissection.
In this study, radiation treatment was only used for the limited dissection group and local recurrence in this group was not significantly lower than that of the total dissection group (14)

Ahlgren et al. showed that performing the limited axillary lymph node dissection (LALND) using the appropriate technique has $97.3 \%$ sensitivity and 98.5\% NEG.PRED.VAL (15).

Moreover, Stewart et al. have revealed that DFS and OS were not affected in patients at stages I and II where axillary lymph node sampling was negative and no additional surgery was done. Of course, a non-significant survival benefit was seen in those patients who received RT after surgery. Accordingly, this shows the importance of performing adjuvant RT in patients with breast cancer (16).

After introducing sentinel lymph node (SLN) and its biopsy for LN negative patients, instead of lymph nodes dissection, the limited dissection became more prevalent. Moreover, the nature of the lymphatic route can be better realized by performing scintigraphy. Chiao et al. showed that the most prevalent place of sentinel node, where the lymph firstly drains to it, is a place between the major pectoralis muscle edge and midaxillary line, which is $2 \mathrm{~cm}$ lower than the hair growth line (17). Accordingly, this place corresponds to the place of beginning of lymph node dissection, which has been referred to in some previous studies performed on the limited axillary dissection.

Various studies have shown that SLNB can be considered a good substitute for axillary lymph node dissection (18-22).

However, there is no consensus for patients with clinically lymph node-positive with or without neoadjuvant chemotherapy, which is still recommended in guidelines. In this regard, it is suggested to remove at least 10 lymph nodes from levels I and II of axillary lymph nodes (7, 23-28).

In SENTINA and Z1071 trials, the role of sentinel node biopsy was examined in patients with positive lymph nodes, during which patients received neoadjuvant chemotherapy. They have revealed that the use of dual trace and removal of at least 3 lymph nodes is effective in decreasing false negative to lower than 10\% (29-34).

It can be concluded that the wide-range axillary lymph nodes dissection is inevitable regarding its outcomes and results. In this regard, it was shown that targeted limited dissection has good therapeutic results even when there is no access to SLNB or when the patient does not want to do it (14). In our center, the limited axillary lymph nodes surgery was done on the selected patients in 27 years (Since 1991). 


\section{Objectives}

Herein, we evaluated DFS as the primary outcome and OS as the secondary outcome based on the number of lymph nodes removed from axilla in the early stage breast cancer patients to achieve the appropriate number of lymph nodes that should be removed when it is not possible to do sentinel lymph node biopsy (SLNB) (there is no access to it) and/or being a contraindication to do it for evaluating the status of axillary lymph nodes.

\section{Methods}

The current research was a large retrospective crosssectional study conducted by reviewing the files of patients with breast cancer at the Cancer Research Center of Shahid Beheshti University of Medical Sciences from 1984 to 2019.

All patients with breast cancer, who were at the early stage of breast cancer with no distant metastasis and/or wide-range involvement of axillary lymph nodes during diagnosis $\left(\mathrm{N}_{3}\right)$, were enrolled in this study. All the patients, who received the systematic neo-adjuvant treatment and all those whose axillary lymph nodes' evaluations were done using the SLNB method, were excluded from the present study.

\subsection{Axillary Lymph Nodes' Examination Method}

Two surgery methods have been used for the patients including: 1- Axillary lymph node dissection (ALND), removing at least 9 lymph nodes of levels I and II of axillary lymph nodes was done for these patients; 2 - The LALND, In this method, lymph node dissection was done by opening clavicle-pectoral fascia from the major pectoralis muscle edge toward the back and up to the intercostobrachial neural branch, and the axillary vein was not explored (removing 1 - 8 lymph nodes).

\subsection{Grouping}

Based on the number of lymph nodes counted in pathology, the patients were divided into 4 groups ( $\mathrm{N}: 1$ $4, N: 5-6, N: 7-8$, and $N \geq 9$ ). Thereafter, based on the number of the lymph nodes involved, the patients were divided into 3 subgroups $\left(\mathrm{N}_{0}\right.$ : 0 involved node, $\mathrm{N}_{1}: 1$ - 3 involved nodes, and $\mathrm{N}_{3}: 4$ - 9 involved nodes).

\subsection{Collecting Information}

Patients' information included age, disease stage, tumor size, number of the lymph nodes removed, and number of the positive lymph nodes. Moreover, biological characteristics of tumors including the type of histology, cell grade, ER, PR, HER2, and LVI were also collected. Afterward, the patients were grouped into $\leq 35,35-39,40-49,50$ - 59, 60 - 69, $70-79$, and $\geq 80$-year-old age groups. It is noteworthy that complementary treatments including radiotherapy, chemotherapy, and hormone therapy were extracted. Follow-up information was examined and, then, updated by clinical visits or phone calls. In the present study, local (In-breast) recurrence, axillary recurrence, and distant metastasis were considered the primary outcomes, and death was considered the secondary outcome. In the cases of recurrence and metastasis in a patient, the first event was entered into the calculations. However, all the events were recorded in statistics.

In the current study, DFS was defined from the diagnosis of disease to the occurrence of the first event (recurrence or metastasis) and OS was defined from the diagnosis time to death.

\subsection{Calculations}

Using SPSS 21 software, the first modeling was done and after calculating the frequency of demographic characteristics of the patients, Kaplan-Mayer Curve was obtained for DFS and OS in different groups. Subsequently, the adjusted hazard ratio (AHR) was calculated and, then, compared using the COX model and by considering some factors affecting recurrence and death.

\subsection{Ethics}

All procedures performed in our study followed the ethical standards of the institution and/or national research committee and with the 1964 Helsinki Declaration and its later amendments or comparable ethical standards.

The Ethics Committee of Shahid Beheshti University of Medical Sciences approved this study in 2019-11-12 (ID: IR.SBMU.CRC.REC.1398.022).

\section{Results}

Of 4436 patient files reviewed, 1644 met the inclusion criteria of this study, of whom 8 patients were men and the others were women. In the current study, the mean followup was $210 \pm 13.6$ months, which was not significantly different among the different groups $(\mathrm{P}<0.05)$. 
The age of the patients ranged from 17 to 87 years old $(49.08 \pm 11.58)$. Regarding the age grouping, the highest number of the patients were in the 40 to 49 -year-old group (31.3\%). The demographic and biological characteristics of the patients are presented in Table 1.

The results presented in Table 2 and Figures 1 - 3 reveal no significant difference among the groups in terms of DFS $(\mathrm{P}=0.268,0.123$, and 0.333$)$.

Besides, in terms of OS, the results revealed that OS was significantly lower only in patients who had 1 - 3 involved lymph nodes and 1 - 4 removed lymph nodes (N:1 - 4, N+: 1 $3)$ compared to the others. These findings are presented in Table 3 and Figures 4 - 6.

Moreover, based on the obtained results, the AHR in the COX model was calculated and, then, compared in the groups. We have also found that AHR was higher in the patients who had lymph node involvement with 1 - 4 removed lymph nodes compared to others, which is shown in Table 4.

In Table 5, the relationship between adjuvant radiotherapy and events such as local recurrence, distant metastases, and death is indicated.

\section{Discussion}

The current study was a single-institute experience on the results of axillary surgery at the early stage of breast cancer, which aimed at determining the effect of the number of removed lymph nodes on DFS and OS during 210 months of follow-up. Historically, axillary surgery has always been an integral part of the treatment of breast cancer.

Concerning some major changes made in attitudes toward the role of the lymphatic system, especially in breast cancer, its therapeutic role is not currently considered for ALND, and the involvement of these nodes is known as a valuable prognostic factor. Various studies have shown that the removal of a higher number of axillary lymph nodes does not increase DFS and OS. Moreover, it was found that sentinel node biopsy can be considered a good substitute for ALND in patients at the early stage of the disease.

However, the levels I and II anatomic ALND is currently the preferred procedure for axillary assessment for correct staging under the conditions, where the limited palpable or detectable lymph nodes in radiology, lack of access to the required technology for SLNB, or lack of desire to receive radioactive material or colored drug by patients. The impact of the number of the resected axillary lymph nodes on survival is still unclear. In this regard, some retrospective reports suggested inferior survival with fewer (often <
10) resected ALNs (7, 23-28). Accordingly, this high volume of lymph node removal is accompanied by more complications, especially in those cases that axillary lymph nodes are not involved. Thus, the current study was performed based on axillary lymph node surgery to realize the lymphatic system of the breast and to determine the accuracy of staging with limited axillary dissection and the removal of less than 10 lymph nodes.

Under these conditions, removing lymph nodes in classic form wherein levels I and II are removed, is more than what is needed. Therefore, the LALND idea has been under debate for many years. In various studies, it has been shown that the axillary lymph node is the main place of breast lymphatic drainage and thus metastasis. In this route, drainage is firstly done into lymph nodes level I, then to level II, and finally to level III. However, bypass of this route and a higher-level involvement limitedly occurs with no lower-level involvement or skip lesion, which can be up to $2 \%(11,12)$. After the formation of scintigraphy and performing SLNB, these routes can be better realized. In a study, it was shown that the most prevalent place of sentinel node is between the major pectoralis edge and MAL, which is $2 \mathrm{~cm}$ lower than the hair growth line. Thus, the beginning of dissection from here can theoretically contain some potentially involved nodes. Similarly, a study was conducted to confirm the place of axillary lymph nodes as well as determining the value of the LALND, which showed that performing LALAND using the appropriate technique has a sensitivity of $97.3 \%$ and NEG.PRED.VAL of $98.5 \%$ (17).

In the current study, two big groups of patients (the ALND group with removing more than 9 lymph nodes and the LALAND group with 3 subgroups of $1-4,5-6$, and $7-8$ lymph nodes) and 3 subgroups in terms of the lymph node involved $\left(\mathrm{N}_{0}: 0, \mathrm{~N}_{1}: 1-3\right.$, and $\mathrm{N}_{2}: 4$ - 9) were examined and, then, compared in terms of the effect of the number of the removed lymph nodes on OS and DFS. Finally, the results showed no significant difference in terms of OS among the patients with no lymph node involved $(\mathrm{AHR}=1.78,1.92$, and $1.2)$.

Accordingly, increasing the number of lymph nodes removed did not result in a significant survival benefit. Moreover, in the patients with the limited involvement of axillary lymph nodes $\left(\mathrm{N}_{1}\right)$, no significant difference was observed in OS between the groups of N:7-8, N: $5-6$, and N $\geq 9$. However, in the group N:7-8, OS was better than the group N: 5 - $6(A H R=1.6,0.24)$. Moreover, this value was significantly higher in the group N: 1 - 4 compared to the two other groups $(\mathrm{AHR}=2.7)$, which shows that in the case of lymph node involvement, removing a few numbers of lymph nodes could consequently result in leaving residual 


\begin{tabular}{|c|c|c|c|c|c|c|c|c|c|c|c|}
\hline \multirow{2}{*}{ Groups/N+ } & \multicolumn{2}{|c|}{$N=1 \cdot 4$} & \multicolumn{3}{|c|}{$N=5-6$} & \multicolumn{3}{|c|}{$N=7 \cdot 9$} & \multicolumn{3}{|c|}{$\mathbf{N} \geq \mathbf{9}$} \\
\hline & $\mathrm{N}_{\mathbf{0}}$ & $\mathrm{N}_{1}$ & $\mathrm{~N}_{\mathbf{0}}$ & $\mathrm{N}_{1}$ & $\mathrm{~N}_{2}$ & $\mathrm{~N}_{\mathbf{0}}$ & $\mathbf{N}_{\mathbf{1}}$ & $\mathrm{N}_{2}$ & $\mathrm{~N}_{\mathbf{0}}$ & $\mathbf{N}_{1}$ & $\mathrm{~N}_{\mathbf{2}}$ \\
\hline \multicolumn{12}{|l|}{ Age } \\
\hline$<35$ & 2 & 2 & 18 & 5 & 0 & 18 & 7 & 1 & 45 & 35 & 7 \\
\hline $35-39$ & 1 & 0 & 10 & 7 & 0 & 17 & 9 & 0 & 53 & 43 & 9 \\
\hline $40-49$ & 6 & 2 & 56 & 22 & 5 & 56 & 34 & 6 & 176 & 133 & 203 \\
\hline $50-59$ & 6 & 5 & 43 & 15 & 1 & 47 & 23 & 6 & 124 & 90 & 16 \\
\hline $60-69$ & 4 & 2 & 14 & 16 & 0 & 23 & 13 & 4 & 62 & 33 & 7 \\
\hline $70-79$ & 0 & 1 & 12 & 2 & 1 & 9 & 6 & 0 & 21 & 18 & 0 \\
\hline$\geq 80$ & 0 & 1 & 1 & 2 & 0 & 1 & 0 & 0 & 8 & 1 & 1 \\
\hline \multicolumn{12}{|l|}{ Cytology } \\
\hline IDC & 17 & 11 & 145 & 73 & 8 & 154 & 376 & 61 & 484 & 549 & 85 \\
\hline ILC & 2 & 1 & 14 & 7 & 0 & 20 & 21 & 4 & 32 & 36 & 5 \\
\hline \multicolumn{12}{|l|}{ Stage } \\
\hline I & 12 & 0 & 66 & 0 & 0 & 88 & 2 & 0 & 203 & 12 & 4 \\
\hline II & 8 & 12 & 99 & 79 & 7 & 99 & 92 & 15 & 346 & 382 & 53 \\
\hline \multicolumn{12}{|l|}{ ER } \\
\hline POS & 18 & 9 & 107 & 62 & 7 & 119 & 74 & 12 & 320 & 260 & 38 \\
\hline \multicolumn{12}{|l|}{ PR } \\
\hline POS & 17 & 9 & 104 & 62 & 7 & 105 & 68 & 11 & 310 & 242 & 35 \\
\hline \multicolumn{12}{|l|}{ ER2 } \\
\hline POS & 3 & 3 & 26 & 13 & 4 & 36 & 14 & 4 & 98 & 75 & 10 \\
\hline \multicolumn{12}{|l|}{ Grade } \\
\hline 1 & 4 & 1 & 22 & 4 & 0 & 25 & 13 & 1 & 69 & 35 & 1 \\
\hline 2 & 11 & 5 & 82 & 46 & 6 & 87 & 57 & 11 & 251 & 215 & 31 \\
\hline 3 & 4 & 5 & 43 & 21 & 0 & 45 & 25 & 2 & 145 & 114 & 23 \\
\hline \multicolumn{12}{|l|}{ ADJ-RT } \\
\hline Yes & 20 & 11 & 136 & 65 & 7 & 146 & 83 & 15 & 385 & 303 & 46 \\
\hline No & 0 & 1 & 38 & 15 & 1 & 45 & 15 & 4 & 186 & 100 & 22 \\
\hline
\end{tabular}

Abbreviations: LVI, lymphovascular invasion; ADJ-RT, adjuvant radiotherapy.

untreated axillary disease.

In $\mathrm{N}_{2}$, which had more than 3 involved lymph nodes, no significant difference was seen in terms of OS among N: 5 $6, \mathrm{~N}: 7-8$, and $\mathrm{N} \geq 9(\mathrm{AHR}=1.21,1.66)$. In this group of patients, there was no case of dissection lower than 5 lymph nodes (Group N:1 - 4).

In the Edinburg trial, the limited axillary dissection and axillary clearance were compared in terms of DFS and OS. In this study, the total ALND of levels I and II (and performing no adjuvant radiotherapy) was done only in one group, and the LALND in the other group was done at the lower level of the tail of the breast toward the back (and performing no adjuvant radiotherapy) (14). Also, local recurrence was a bit higher in the total dissection group; however, this difference was not significant. Besides, no significant difference was seen in terms of OS between these two groups.

In this study, the patients in the total dissection group received no adjuvant radiotherapy and 10-year survival was about $60 \%$. However, in the current study, $70 \%$ of the patients in the $\mathrm{N} \geq 9$ group and $80 \%$ of the subjects of the other groups (the LALND) received adjuvant radiotherapy, and 10 -year survival was more than $80 \%$. Accordingly, this difference can confirm the importance of adjuvant radiotherapy for improving OS.

It can be concluded that the LALND with removing less than 9 nodes does not worsen the OS compared to axillary clearance, which may indicate the correct staging.

In the current study, there was no difference in terms of DFS in the group $\mathrm{N}_{0}(\mathrm{AHR}=1.43,1.76,1.74)$. Also, there were no significant differences in DFS and AHR in the group $\mathrm{N}_{1}(\mathrm{AHR}=1.74,0.97,0.28)$. However, because of the small number of the samples in the group $\mathrm{N}_{2}$, DFS and AHR were not comparable (AHR: N/E).

Generally, OS and DFS were in a good range accompanied by the minimum increased risk in the limited dissec- 


\begin{tabular}{|c|c|c|c|}
\hline & Total & Recurrence or Metastases & No Events, No. (\%) \\
\hline \multicolumn{4}{|l|}{$\mathbf{N}+=\mathbf{0}$} \\
\hline$N=1-4$ & 20 & 2 & $18(90.0)$ \\
\hline$N=5,6$ & 171 & 16 & $155(90.6)$ \\
\hline $\mathrm{N}=7,8$ & 186 & 12 & $174(93.5)$ \\
\hline$N \geq 9$ & 563 & 33 & $530(94.1)$ \\
\hline Overall & 940 & 63 & $877(93.3)$ \\
\hline \multicolumn{4}{|l|}{$\mathbf{N}+=\mathbf{1}-\mathbf{3}$} \\
\hline$N=1-4$ & 12 & 1 & $11(91.7)$ \\
\hline$N=5,6$ & 80 & 9 & $71(88.8)$ \\
\hline$N=7,8$ & 93 & 2 & $91(97.8)$ \\
\hline$N \geq 9$ & 398 & 34 & $364(91.5)$ \\
\hline Overall & 583 & 46 & $537(92.1)$ \\
\hline \multicolumn{4}{|l|}{$N+=4-9$} \\
\hline$N=5,6$ & 8 & 0 & $8(100.0)$ \\
\hline $\mathrm{N}=7,8$ & 18 & 1 & $17(94.4)$ \\
\hline$N \geq 9$ & 65 & 11 & $54(83.1)$ \\
\hline Overall & 91 & 12 & $79(86.8)$ \\
\hline
\end{tabular}

Table 3. Overall Survival

\begin{tabular}{|c|c|c|c|}
\hline & Total & Death & No Events, No. (\%) \\
\hline \multicolumn{4}{|l|}{$\mathbf{N}+=\mathbf{0}$} \\
\hline$N=1-4$ & 20 & 1 & $19(95.0)$ \\
\hline $\mathrm{N}=5,6$ & 173 & 11 & $162(93.6)$ \\
\hline$N=7,8$ & 190 & 11 & $179(94.2)$ \\
\hline$N \geq 9$ & 570 & 30 & $540(94.7)$ \\
\hline Overall & 953 & 53 & $900(94.4)$ \\
\hline \multicolumn{4}{|l|}{$\mathbf{N}+=\mathbf{1}-\mathbf{3}$} \\
\hline$N=1-4$ & 12 & 3 & $9(75.0)$ \\
\hline $\mathrm{N}=5,6$ & 80 & 4 & $76(95.0)$ \\
\hline$N=7,8$ & 95 & 2 & $93(97.9)$ \\
\hline$N \geq 9$ & 401 & 22 & $379(94.5)$ \\
\hline Overall & 588 & 31 & $557(94.7)$ \\
\hline \multicolumn{4}{|l|}{$N+=4-9$} \\
\hline$N=5,6$ & 8 & 1 & $7(87.5)$ \\
\hline $\mathrm{N}=7,8$ & 19 & 1 & $18(94.7)$ \\
\hline$N \geq 9$ & 65 & 6 & $59(90.8)$ \\
\hline Overall & 92 & 8 & $84(91.3)$ \\
\hline
\end{tabular}




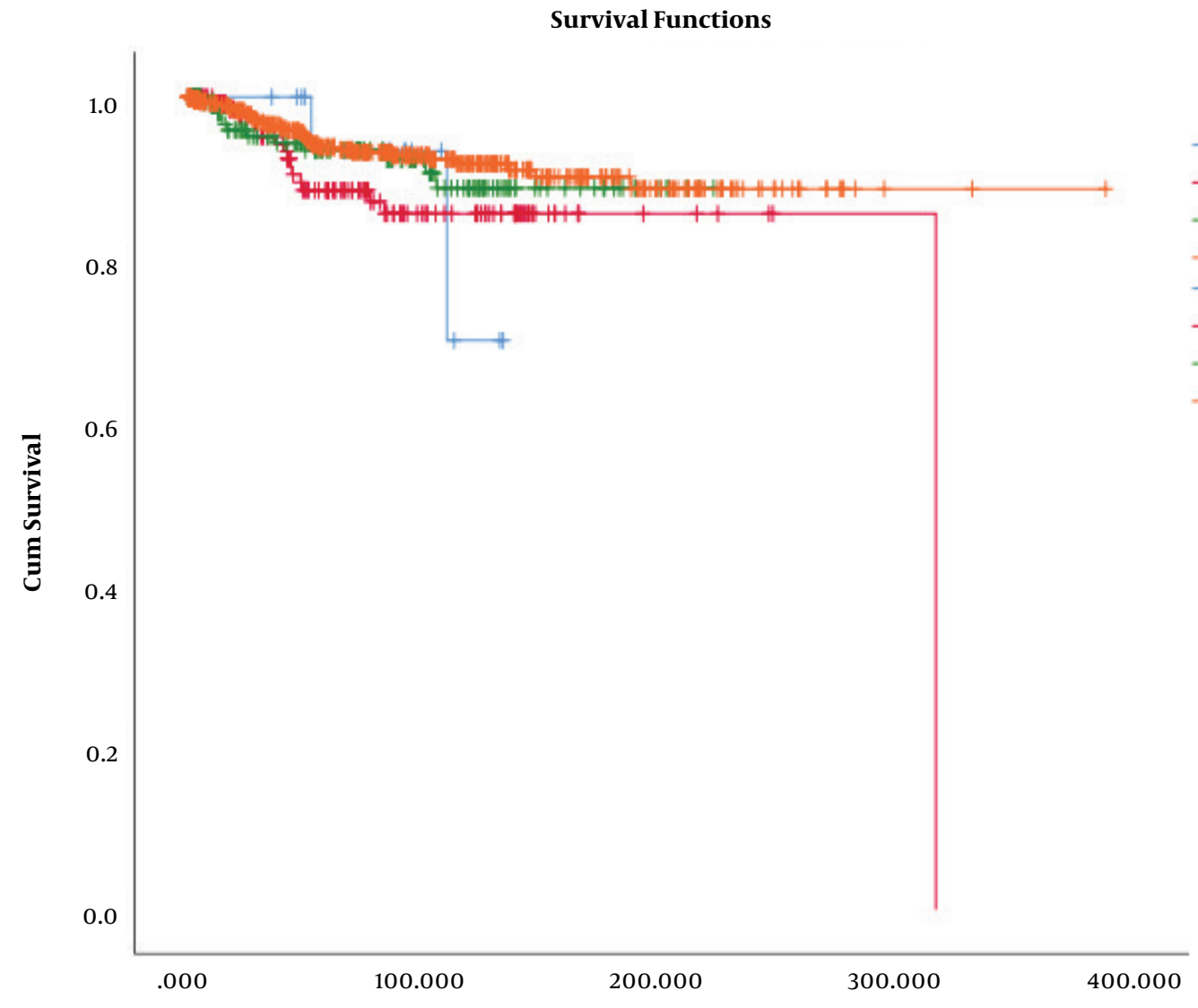

N_cat

$\neg \mathrm{N}=1-4$

$\neg \mathrm{N}=5,6$

$\neg \mathrm{N}=7,8$

$\neg \mathrm{N}>=9$

$+\mathrm{N}=1$-4-censored

$+\mathrm{N}=5,6$-censored

$+\mathrm{N}=7,8$-censored

† $\mathrm{N}>=9$-censored

Time To Recurrence

\begin{tabular}{|c|c|c|c|c|}
\hline \multicolumn{5}{|c|}{ Figure 1. Kaplan-Mayer curve in the group N+: 0 (DFS) } \\
\hline & $\mathbf{N}+=\mathbf{0} \& \mathbf{N} \geq \mathbf{9}$ & $N+=0 \& N=7,8$ & $N+=0 \& N=5,6$ & $\mathbf{N}+=0 \& \mathbf{N}=1-4$ \\
\hline Recurrence AHR & Baseline category & 1.2 & 1.92 & 1.78 \\
\hline \multirow[t]{2}{*}{ Death AHR1 } & Baseline category & 1.74 & 1.76 & 1.43 \\
\hline & $\mathbf{N}+=\mathbf{1}-\mathbf{3} \& \mathbf{N} \geq \mathbf{9}$ & $N+=1-3 \& N=7,8$ & $N+=1-3 \& N=5,6$ & $N+=1-3 \& N=1-4$ \\
\hline Recurrence HR & Baseline category & 0.28 & 0.97 & 1.74 \\
\hline \multirow[t]{2}{*}{ Death AHR } & Baseline category & 0.24 & 1.6 & 2.7 \\
\hline & $\mathrm{N}+=4-9 \& N \geq 9$ & $\mathrm{~N}+=4-9 \& \mathrm{~N}=7,8$ & $N+=4-9 \& N=5,6$ & $N+=4-9 \& N=1-4$ \\
\hline Recurrence AHR & Baseline category & $\mathrm{NE}$ & $\mathrm{NE}$ & - \\
\hline
\end{tabular}

Abbreviation: AHR, adjusted hazard ratio.

tion group by removing 7 - 8 nodes. The main principle in all these cases was observing all complementary standard treatments after the operation including radiotherapy.

In the current study, all the patients who underwent BCS received adjuvant radiotherapy. In the groups that underwent limited axillary dissection and lymph nodes were involved, the majority of the patients received adjuvant radiotherapy (N:1-4=91\%, N:5-6=81\%, and N:7-8=84\%). This value was $74 \%$ for wide-range axillary dissection and recurrence was only observed in one case, who was in the total dissection group $(\mathrm{N} \geq 9)$ with 6 involved lymph nodes and had refused adjuvant RT. In the other groups, in which the 


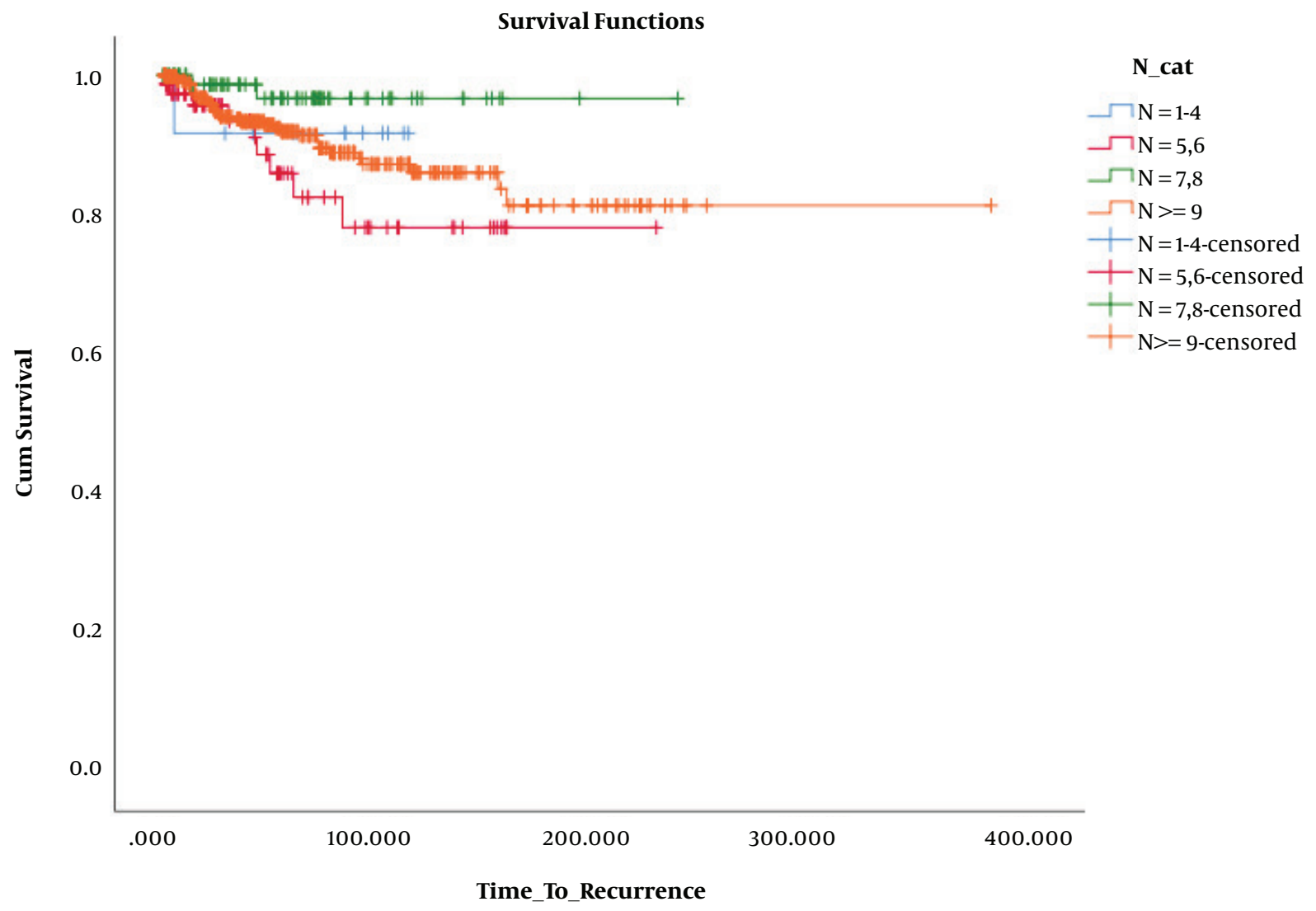

Figure 2. Kaplan-Mayer curve in the group N+: 1-3 (DFS)

limited axillary dissection was done, with or without axillary lymph node involvement, no case of recurrent was reported. However, in the Edinburg study, axillary recurrent was seen for both total ALND and LALND [6 cases (3\%) and 11 cases (5.4\%), respectively]. A comparison of these values showed the importance of performing complementary radiotherapy in improving the outcome for patients with breast cancer.

Various studies have shown that radiotherapy can be considered a substitute for total dissection even with the limited involvement of lymph nodes $(35,36)$. Under such conditions, LALND can be known as an appropriate substitute for the traditional ALND at the early stages of breast cancer.

\subsection{Conclusions}

Regarding the results of the current study as well as comparing it with other studies and concerning adjuvant treatments, age range, and biology of cancer, it can be concluded that performing the LALND in the defined anatomic range and removing 7-8 lymph nodes instead of removing 10 lymph nodes are not inferior when it is not possible to do SLNB (there is no access to it) and/or being a contraindication to do it for evaluating the status of axillary lymph nodes in the patients at the early stage of breast cancer.

We generally believed that in patients who have no palpable or detectable lymph nodes and SLNB is not considered (due to any reason), performing the LALND with removing at least 4 - 6 nodes from the edge of pectoralis major muscle toward the posterior axillary wall containing the external mammary group is acceptable.

However, in cases with axillary lymph node involvement, which SLNB is not recommended, removing at least 7 - 8 lymph nodes is necessary.

Moreover, to decrease the complications of ALND, it is recommended to repeat this study for the patients who have received neoadjuvant chemotherapy. 


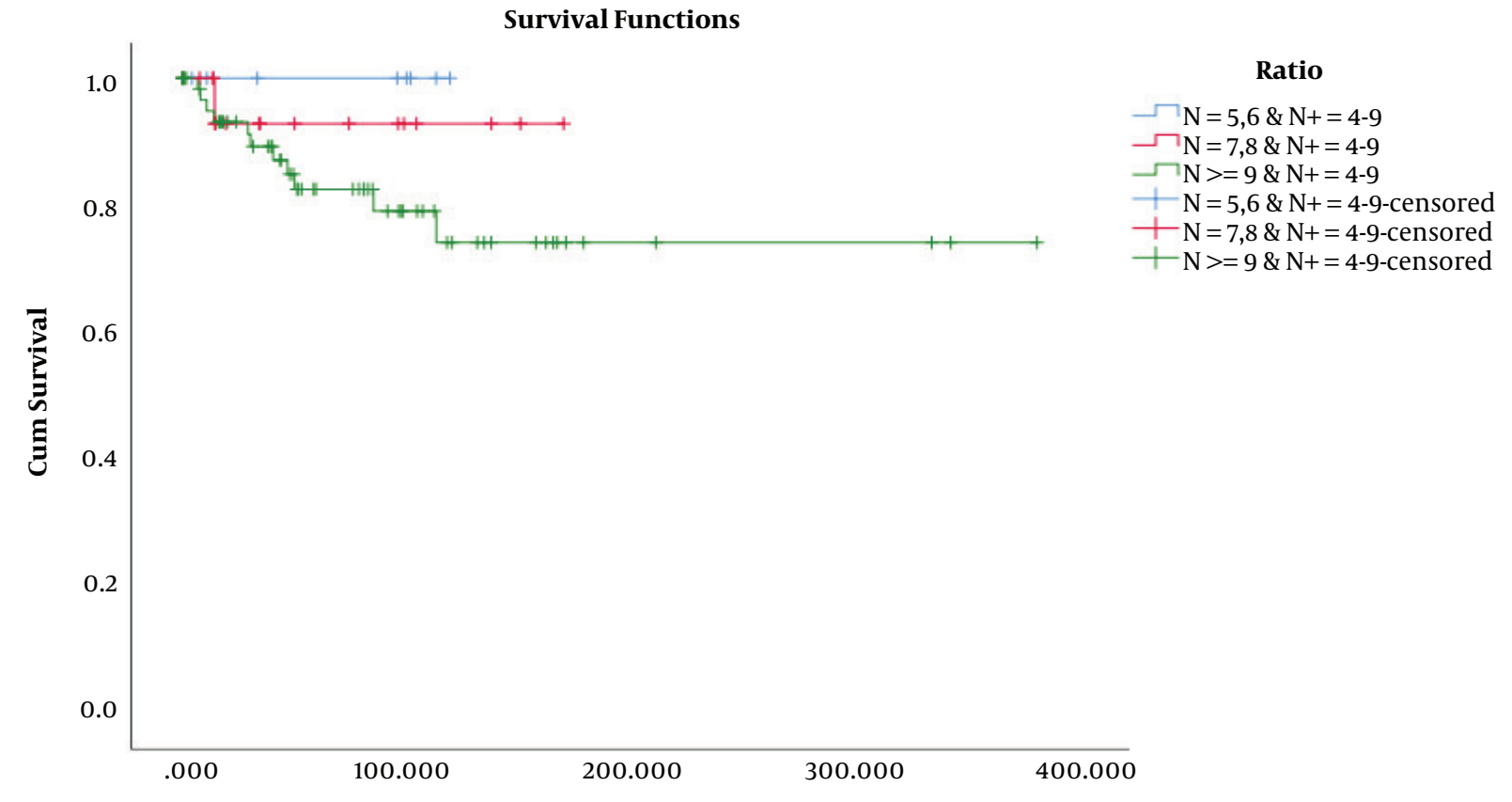

Time_To_Recurrence

Figure 3. Kaplan-Mayer curve in the group N+: 4 - 9 (DFS)

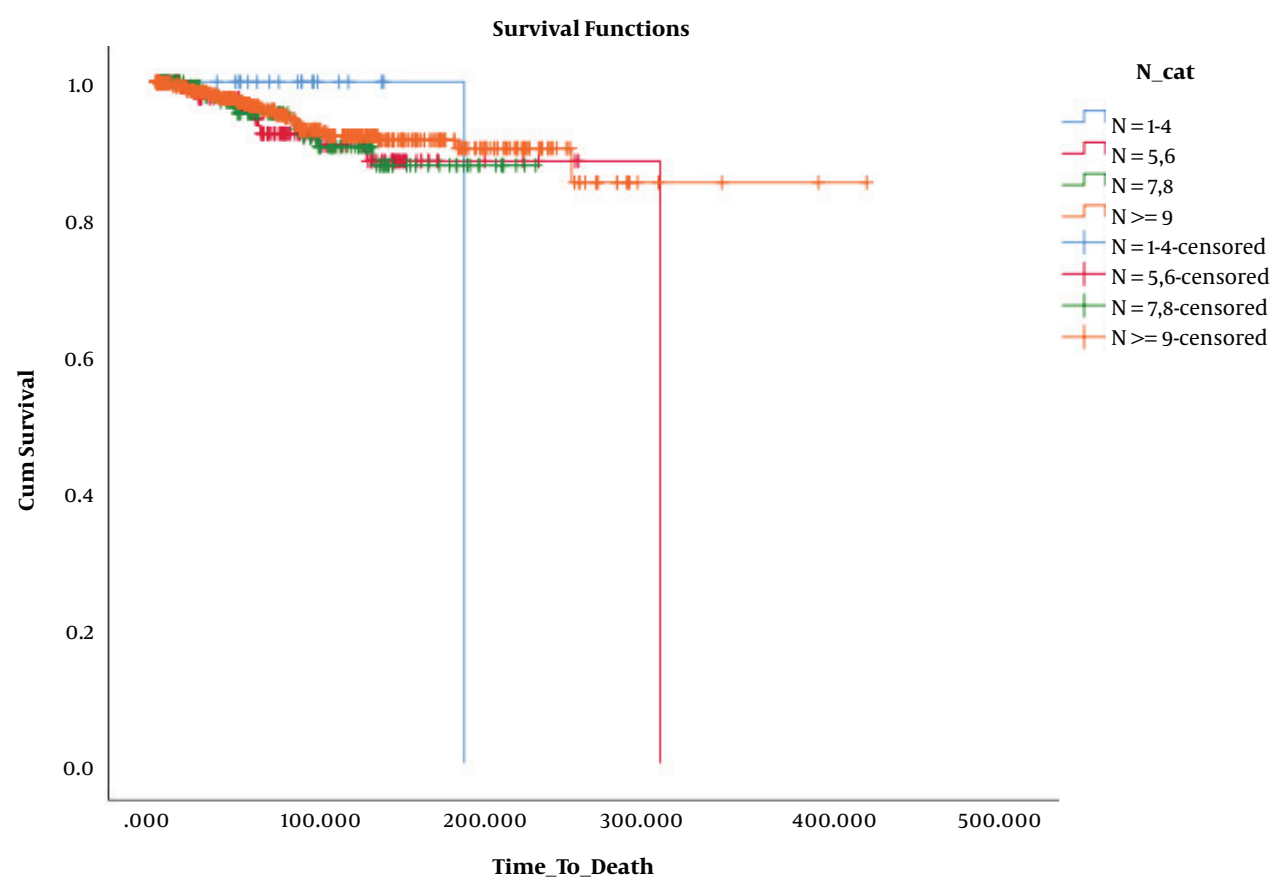

Figure 4. Overall survival in the group N+: 0 


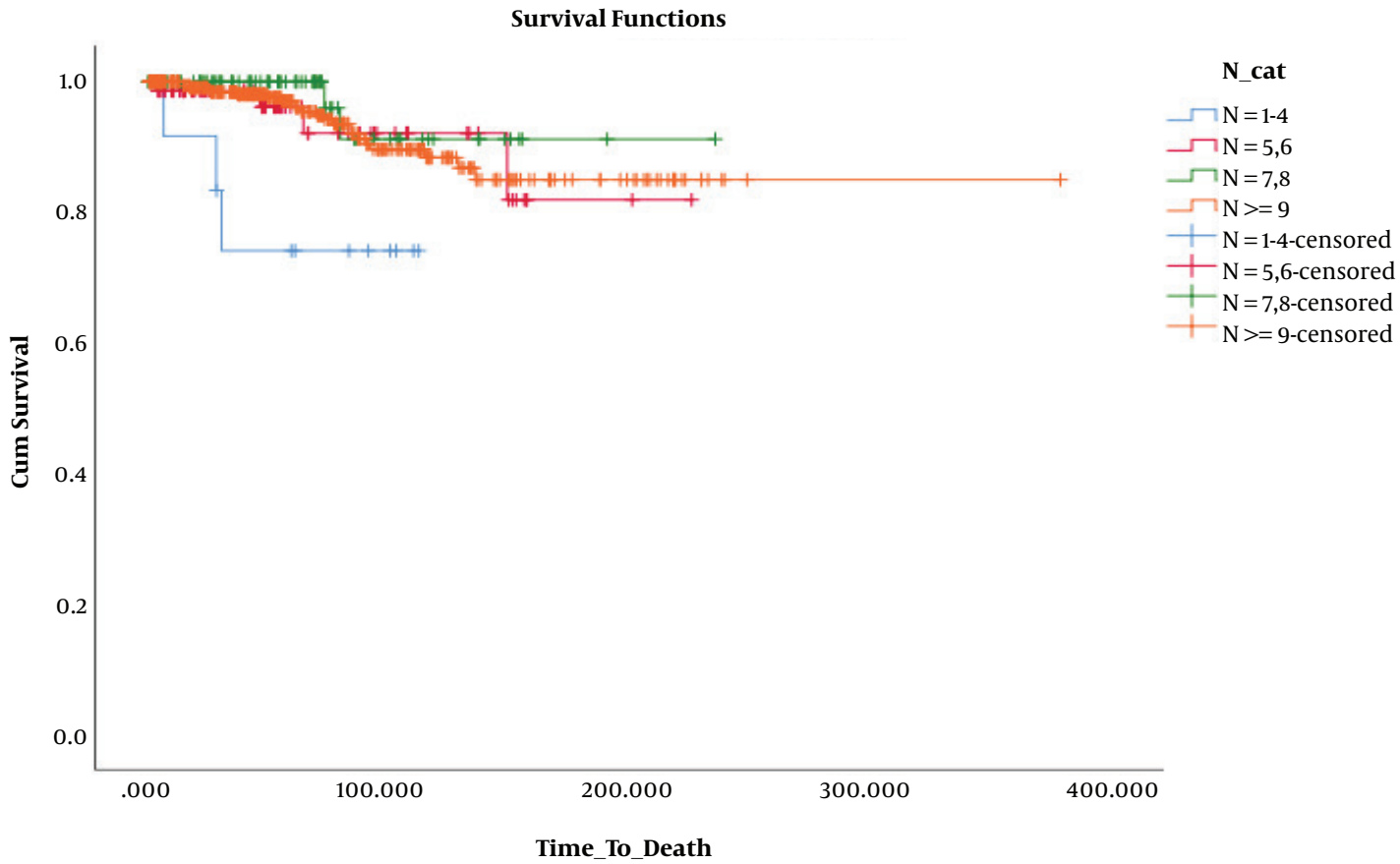

Figure 5. Overall survival in the group N+:1-3

\begin{tabular}{|c|c|c|c|c|c|c|c|c|}
\hline & \multicolumn{4}{|c|}{ Yes } & \multicolumn{4}{|c|}{ No } \\
\hline & $\mathbf{N} / \mathbf{E}$ & IBLR & AxR & DMet & N/E & IBLR & AxR & DMet \\
\hline \multicolumn{9}{|l|}{$\mathbf{N}=\mathbf{1}-\mathbf{4}$} \\
\hline $\mathrm{N}_{0}$ & 18 & 1 & 0 & 1 & 0 & 0 & 0 & 0 \\
\hline N1 & 9 & 0 & 0 & 2 & 1 & 0 & 0 & 0 \\
\hline N2 & - & - & - & - & - & - & - & - \\
\hline \multicolumn{9}{|l|}{$N=5-6$} \\
\hline No & 124 & 4 & 0 & 8 & 31 & 1 & 0 & 6 \\
\hline N1 & 55 & 1 & 0 & 8 & 13 & 1 & 0 & 2 \\
\hline N2 & 6 & 0 & 0 & 1 & 1 & 0 & 0 & 0 \\
\hline \multicolumn{9}{|l|}{$N=7-8$} \\
\hline No & 131 & 4 & 0 & 11 & 40 & 2 & 0 & 3 \\
\hline N1 & 75 & 1 & 0 & 7 & 15 & 0 & 0 & 0 \\
\hline N2 & 13 & 0 & 0 & 2 & 4 & 0 & 0 & 0 \\
\hline \multicolumn{9}{|l|}{$\mathbf{N} \geq \mathbf{9}$} \\
\hline No & 337 & 9 & 9 & 39 & 175 & 0 & 0 & 11 \\
\hline $\mathrm{N} 1$ & 272 & 6 & 0 & 25 & 84 & 1 & 0 & 15 \\
\hline N2 & 40 & 2 & 0 & 4 & 15 & 1 & 1 & 5 \\
\hline
\end{tabular}

Abbreviations: N/E, no event; IBLR, in breast local recurrence; AxR, axillary recurrence; DMet, distant metastases. 


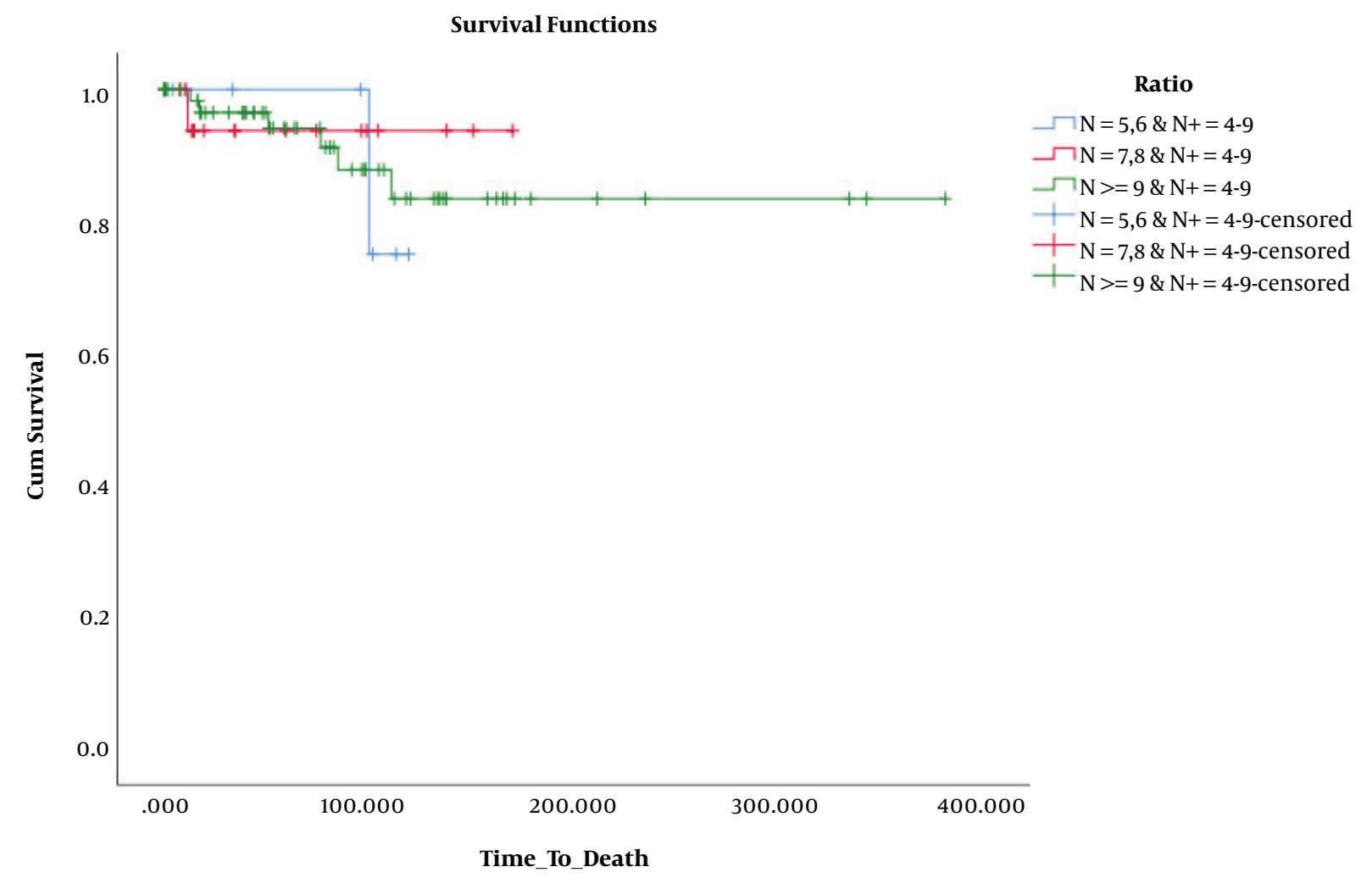

Figure 6. Overall survival in the group N+: 4 - 9

\section{Footnotes}

Authors' Contribution: Study concept and design, Akbari.me,Javadi SMR., and Hashemi S.; Analysis and interpretation of data, Akbari.me,Javadi SMR,Akbari F. M.; Critical revision of the manuscript for important intellectual content, F. M., A,Khayamzadeh M,Moradian F,Mohammadi F; Drafting of the manuscript, Akbari.me,Javadi SMRd .; Statistical analysis, Akbari.me,Javadi SMR., and Hashemi S.

Conflict of Interests: The authors declare that there are no conflicts of interest regarding the publication of this paper.

Ethical Approval: The Ethics Committee of Shahid Beheshti University of Medical Sciences approved this study in 2019-11-12 (ID: IR.SBMU.CRC.REC.1398.022).

Funding/Support: This paper funded by Cancer Research Center of Shahid Beheshti University of Medical Sciences

\section{References}

1. Veronesi U, Paganelli G, Galimberti V, Viale G, Zurrida S, Bedoni M, et al. Sentinel-node biopsy to avoid axillary dissection in breast cancer with clinically negative lymph-nodes. Lancet. 1997;349(9069):1864-7. doi: 10.1016/S0140-6736(97)01004-0. [PubMed: 9217757].

2. Wood WC. Progress from clinical trials on breast cancer. Cancer. 1994;74(9 Suppl):2606-9. doi: 10.1002/10970142(19941101)74:9+<2606::aid-cncr2820741803>3.0.co;2-z. [PubMed: 7954272].

3. Halsted WS. I. The results of operations for the cure of cancer of the breast performed at the Johns Hopkins hospital from June, 1889, to January, 1894. Ann Surg. 1894;20(5):497-555. doi: 10.1097/00000658-189407000-00075. [PubMed: 17860107]. [PubMed Central: PMC1493925].

4. Halsted WS. I. The results of radical operations for the cure of carcinoma of the breast. Ann Surg. 1907;46(1):1-19. doi: 10.1097/00000658190707000-00001. [PubMed: 17861990]. [PubMed Central: PMC1414357].

5. Senofsky GM, Moffat FJ, Davis K, Masri MM, Clark KC, Robinson DS, et al. Total axillary lymphadenectomy in the management of breast cancer. Arch Surg. 1991;126(11):1336-42. doi: 10.1001/archsurg.1991.01410350026004. [PubMed:1747046].

6. Camp RL, Rimm EB, Rimm DL. A high number of tumor free axillary lymph nodes from patients with lymph node negative breast carcinoma is associated with poor outcome. Cancer. 2000;88(1):108-13. [PubMed: 10618612].

7. Joslyn SA, Konety BR. Effect of axillary lymphadenectomy on breast carcinoma survival. Breast Cancer Res Treat. 2005;91(1):11-8. doi: 10.1007/s10549-004-6276-7. [PubMed: 15868427].

8. Gao F, He N, Wu PH. The number of tumor-free axillary lymph nodes removed as a prognostic parameter for node-negative breast cancer. 
Chin J Cancer. 2014;33(11):569-73. doi: 10.5732/cjc.014.10056. [PubMed: 25322865]. [PubMed Central: PMC4244320].

9. Sandoughdaran S, Malekzadeh M, Abari ME. Frequency and predictors of axillary lymph node metastases in iranian women with early breast cancer. Asian Pac J Cancer Prev. 2018;19(6):1617-20. doi: 10.22034/APJCP.2018.19.6.1617. [PubMed: 29936787]. [PubMed Central: PMC6103571].

10. Coombs N, Chen W, Taylor R, Boyages J. A decision tool for predicting sentinel node accuracy from breast tumor size and grade. Breast J. 2007;13(6):593-8. doi: 10.1111/j.1524-4741.2007.00507.x. [PubMed: 17983402].

11. Rosen PP, Lesser ML, Kinne DW, Beattie EJ. Discontinuous or "skip" metastases in breast carcinoma. Analysis of 1228 axillary dissections. Ann Surg.1983;197(3):276-83. doi:10.1097/00000658-19830300000006. [PubMed: 6830335]. [PubMed Central: PMC1352730].

12. Veronesi U, Rilke F, Luini A, Sacchini V, Galimberti V, Campa T, et al. Distribution of axillary node metastases by level of invasion. An analysis of 539 cases. Cancer. 1987;59(4):682-7. doi: 10.1002/10970142(19870215)59:4<682::aid-cncr2820590403>3.0.co;2-z. [PubMed: 3802027].

13. Stewart HJ, Everington D, Forrest APM. The Cardiff local therapy trial - results at 20 years. The Breast. 1994;3(1):40-5. doi: 10.1016/09609776(94)90035-3.

14. Forrest AP, Everington D, McDonald CC, Steele RJ, Chetty U, Stewart HJ. The Edinburgh randomized trial of axillary sampling or clearance after mastectomy. Br J Surg. 1995;82(11):1504-8. doi: 10.1002/bjs.1800821118. [PubMed: 8535804].

15. Ahlgren J, Holmberg L, Bergh J, Liljegren G. Five-node biopsy of the axilla: an alternative to axillary dissection of levels I-II in operable breast cancer. EurJ Surg Oncol. 2002;28(2):97-102. doi: 10.1053/ejso.2001.1228. [PubMed: 11884042].

16. Stewart HJ, Jack WJL, Everington D, Forrest APM, Rodger A, McDonald CC, et al. South-east Scottish trial of local therapy in node negative breast cancer. The Breast. 1994;3(1):31-9. doi: 10.1016/09609776(94)90034-5.

17. Lo C, Lee PC, Yen RF, Huang CS. Most frequent location of the sentinel lymph nodes. Asian J Surg. 2014;37(3):125-9. doi: 10.1016/j.asjsur.2014.01.006. [PubMed: 24637184].

18. Veronesi U, Paganelli G, Viale G, Luini A, Zurrida S, Galimberti V, et al. Sentinel-lymph-node biopsy as a staging procedure in breast cancer: Update of a randomised controlled study. Lancet Oncol. 2006;7(12):983-90. doi: 10.1016/s1470-2045(06)70947-0.

19. Wong JH. Sentinel lymphadenectomy in breast cancer: university research tool or community practice? Surg Clin North Am. 2000;80(6):1821-30. doi: 10.1016/s0039-6109(05)70264-4. [PubMed: 11140876].

20. Tafra L. A randomized comparison of sentinel-node biopsy with routine axillary dissection in breast cancer. Women's Oncol Rev. 2011;4(1):67. doi: 10.3109/14733400410001689342.

21. Zervos EE, Badgwell BD, Abdessalam SF, Farrar WB, Walker MJ, Yee LD, et al. Selective analysis of the sentinel node in breast cancer. Am J Surg. 2001;182(4):372-6. doi: 10.1016/s0002-9610(01)00740-1. [PubMed: 11720674]

22. Cipolla C, Graceffa G, Calamia S, Latteri S, Marino MV, Latteri M, et al. Completion axillary lymph node dissection can be avoid in patients with invasive breast cancer and sentinel lymph node micrometastases. Ann Ital Chir. 2017;6. [PubMed: 29134954].

23. Salama JK, Heimann R, Lin F, Mehta N, Chmura SJ, Singh R, et al. Does the number of lymph nodes examined in patients with lymph node-negative breast carcinoma have prognostic significance? Cancer. 2005;103(4):664-71. doi:10.1002/cncr.20830. [PubMed: 15641038].

24. Sosa JA, Diener-West M, Gusev Y, Choti MA, Lange JR, Dooley WC, et al. Association between extent of axillary lymph node dissection and survival in patients with stage I breast cancer. Ann Surg Oncol. 1998;5(2):140-9. doi: 10.1007/BF02303847. [PubMed: 9527267].

25. Mersin H, Yildirim E, Bulut H, Berberoglu U. The prognostic significance of total lymph node number in patients with axillary lymph node-negative breast cancer. Eur J Surg Oncol. 2003;29(2):132-8. doi 10.1053/ejso.2002.1285. [PubMed: 12633555].

26. Polednak AP. Survival of lymph node-negative breast cancer patients in relation to number of lymph nodes examined. Ann Surg. 2003;237(2):163-7. doi: 10.1097/01.SLA.0000048552.84451.C5. [PubMed: 12560772]. [PubMed Central: PMC1522136].

27. Weir L, Speers C, D'Yachkova Y, Olivotto IA. Prognostic significance of the number of axillary lymph nodes removed in patients with node-negative breast cancer. J Clin Oncol. 2002;20(7):1793-9. doi: 10.1200/JCO.2002.07.112. [PubMed: 11919236].

28. van der Wal BC, Butzelaar RM, van der Meij S, Boermeester MA. Axillary lymph node ratio and total number of removed lymph nodes: predictors of survival in stage I and II breast cancer. Eur J Surg Oncol. 2002;28(5):481-9. doi: 10.1053/ejso.2002.1239. [PubMed: 12217299].

29. Xing Y, Foy M, Cox DD, Kuerer HM, Hunt KK, Cormier JN. Meta-analysis of sentinel lymph node biopsy after preoperative chemotherapy in patients with breast cancer. Br J Surg. 2006;93(5):539-46. doi 10.1002/bjs.5209. [PubMed: 16329089].

30. Kelly AM, Dwamena B, Cronin P, Carlos RC. Breast cancer sentinel node identification and classification after neoadjuvant chemotherapy-systematic review and meta analysis. Acad $\mathrm{Ra}$ diol. 2009;16(5):551-63. doi: 10.1016/j.acra.2009.01.026. [PubMed: 19345896].

31. Boughey JC, Suman VJ, Mittendorf EA, Ahrendt GM, Wilke LG, Taback B, et al. Sentinel lymph node surgery after neoadjuvant chemotherapy in patients with node-positive breast cancer: The ACOSOG Z1071 (Alliance) clinical trial. JAMA. 2013;310(14):1455-61. doi: 10.1001/jama.2013.278932. [PubMed: 24101169]. [PubMed Central: PMC4075763].

32. Kuehn T, Bauerfeind I, Fehm T, Fleige B, Hausschild M, Helms G, et al. Sentinel-lymph-node biopsy in patients with breast cancer before and after neoadjuvant chemotherapy (SENTINA): A prospective, multicentre cohort study. Lancet Oncol. 2013;14(7):609-18. doi: 10.1016/S1470-2045(13)70166-9. [PubMed: 23683750].

33. Boileau JF, Poirier B, Basik M, Holloway CM, Gaboury L, Sideris L, et al. Sentinel node biopsy after neoadjuvant chemotherapy in biopsyproven node-positive breast cancer: The SN FNAC study. J Clin Oncol. 2015;33(3):258-64. doi: 10.1200/JCO.2014.55.7827. [PubMed: 25452445].

34. Henke G, Knauer M, Ribi K, Hayoz S, Gerard MA, Ruhstaller T, et al Tailored axillary surgery with or without axillary lymph node dissection followed by radiotherapy in patients with clinically nodepositive breast cancer (TAXIS): Study protocol for a multicenter, randomized phase-III trial. Trials. 2018;19(1):667. doi: 10.1186/s13063-0183021-9. [PubMed: 30514362]. [PubMed Central: PMC6278139].

35. Donker M, van Tienhoven G, Straver ME, Meijnen P, van de Velde CJ, Mansel RE, et al. Radiotherapy or surgery of the axilla after a positive sentinel node in breast cancer (EORTC 10981-22023 AMAROS): A randomised, multicentre, open-label, phase 3 non-inferiority trial. Lancet Oncol. 2014;15(12):1303-10. doi: 10.1016/S1470-2045(14)70460-7. [PubMed: 25439688]. [PubMed Central: PMC4291166].

36. Giuliano AE, Ballman KV, McCall L, Beitsch PD, Brennan MB, Kelemen $\mathrm{PR}$, et al. Effect of axillary dissection vs no axillary dissection on 10year overall survival among women with invasive breast cancer and sentinel node metastasis: The ACOSOG Z0011 (alliance) randomized clinical trial. JAMA. 2017;318(10):918-26. doi: 10.1001/jama.2017.11470. [PubMed: 28898379]. [PubMed Central: PMC5672806]. 